Archives of Clinical and Medical Case Reports

doi: $10.26502 / \mathrm{acmcr} .96550027$

fortune

Volume 2, Issue 3

Case Report

\title{
A Case with Tumor, Tuberculosis and Chylothorax
}

\author{
Kübra Așık Cansız ${ }^{1}$, Tuncer Tuğ ${ }^{1}$, Suat Konuk ${ }^{1}$, Hacı Ali Kılıçü̈n ${ }^{2}$ and Suphi Aydın ${ }^{3}$ \\ ${ }^{1}$ Abant Izzet Baysal University, Department of Pulmonary Diseases, Bolu, Turkey \\ ${ }^{2}$ Abant Izzet Baysal University, Department of Thoracic Surgery, Bolu, Turkey \\ ${ }^{3}$ Izzet Baysal State Hospital, Department of Chest Surgery, Bolu, Turkey
}

*Corresponding Author: Dr. Suat Konuk, Abant Izzet Baysal University, Faculty of Medicine, Pulmonology Department, Bolu, Turkey, Tel: 90507341 0126; E-mail: suatkonukk@windowslive.com

Received: 19 March 2018; Accepted: 04 April 2018; Published: 01 May 2018

\begin{abstract}
Chylothorax is a rare clinical condition caused by the accumulation of lymphatic fluid in the pleural space. Chylothorax often results in thoracic trauma and malignant obstruction. More rare causes are tuberculosis, infanjiomiyomatosis, venous thrombosis, congenital lymphatic malformation, nephrotic syndrome, hypothyroidism, cirrhosis, chemotherapeutic drugs, sarcoidosis and idiopathic chylothorax. A 77-year-old female patient with a one year history of colon cancer and post-op chemotherapy was referred to us with the suspicion of metastasis upon detection of lymphadenomas and pleural effusion during a thoracic CT in follow-up. Thoracentesis was performed for the pleural effusion of the patient and chylous fluid was aspirated. The diagnosis of chylothorax was confirmed by pleural fluid biochemical analysis. Results were consistent with tuberculosis from the biopsy performed on synchronous lymph nodes. Due to multiple causes in the etiology of chylothorax, we presented our case.
\end{abstract}

Keywords: Chylothorax; Tuberculosis; Malignancy

\section{Introduction}

Chylothorax is a rare clinical condition caused by the accumulation of lymphatic fluid in the pleural space [1]. It was first described by Bartolet in 1633 and was recognized by the milk-like appearance of the effusion [2]. According to a study by Staats in the 1950s, liquids with a triglyceride level above $110 \mathrm{mg} / \mathrm{dl}$ were defined as chylous pleural effusion and this definition still remains valid [3]. The accumulation of lymphatic fluid due to chylothorax may lead 
to severe metabolic and immunological disorders. Chylothorax often results in thoracic trauma and malignant obstruction. More rare causes are tuberculosis, infanjiomiyomatosis, venous thrombosis, congenital lymphatic malformation, nephrotic syndrome, hypothyroidism, cirrhosis, chemotherapeutic drugs, sarcoidosis and idiopathic chylothorax [4]. During the thoracentesis, if the pleural fluid aspirated has a milky white appearance, chylothorax should be considered. Once the diagnosis is confirmed by biochemical methods, treatment should be started immediately [5]. The first step after finding chylothorax is to find the cause of the disease. Conservative treatments together with the treatment of the underlying disorder contribute to the improvement of the disease. Treatment of patients with confirmed chylothorax begins with the drainage of lymphatic fluid from the chest cavity. In some cases this can be accomplished by thoracentesis but tube thoracostomy is necessary in many cases where fluid accumulation is rapid. Thereafter, ductal lymphatic flow reduction measures are taken. Initially, it is ensured that the fatty acids taken orally are medium chain triglycerides which can pass directly to the portal venous system. In necessary cases, oral intake may need to be stopped completely and parenteral nutrition may be required. In recent years, it has been reported that in addition to these conservative interventions, somatostatin and analogs can increase the efficacy of treatment $[6,7]$. Therapeutic approaches such as duct ligation, pleuroperitoneal shunt and pleurodesis come to the fore in cases that can not be controlled by conservative methods. In a study by Cho et al., in 67 cases of chylothorax, total parenteral nutrition were reported to be successful in 24 of 46 patients, 20 cases needed the addition of talc pleurodesis and only two patients required surgical treatment [8].

\section{Case}

A 77-year-old female patient with a 1 year old history of colon cancer and post-op chemotherapy; was referred to us with the suspicion of metastasis upon detection of lymphadenomas and pleural effusion during a thoracic CT in follow-up (Figure 1 and 2). There was occasional cough, no complaints of phlegm or shortness of breath. No night sweats or chest pain. There was a loss of appetite. On physical examination, respiratory sounds in the lower right lung were decreased; no pathology was found in other systemic examinations. BP: 120/70 mm/Hg, Spo2:97 (room air), Pulse:87 beat/min. Biochemical parameters: CRP:0,7 mg/dl ESR:46 mm/h WBC:5.

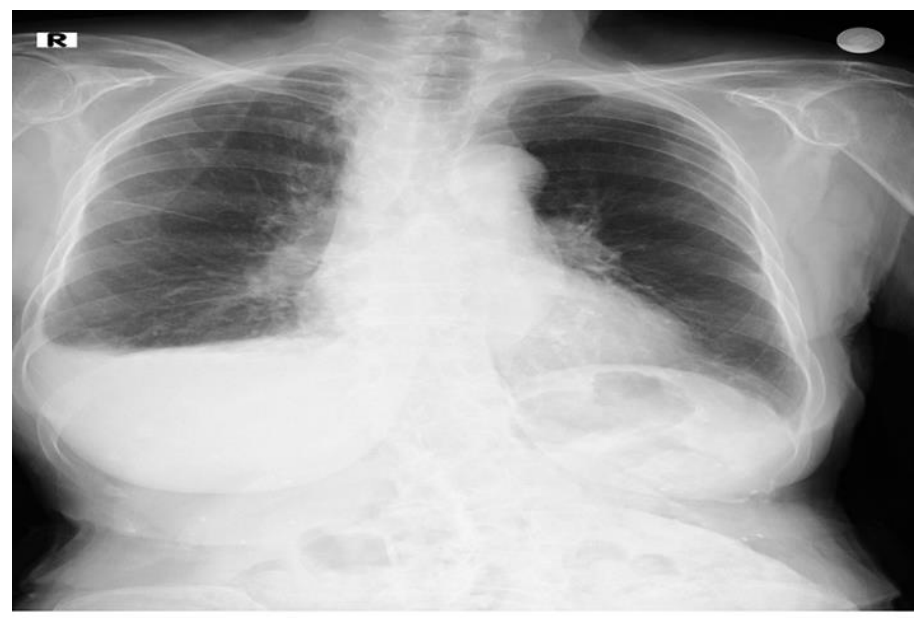

Figure 1: Postero-anterior Lung Graphy. 


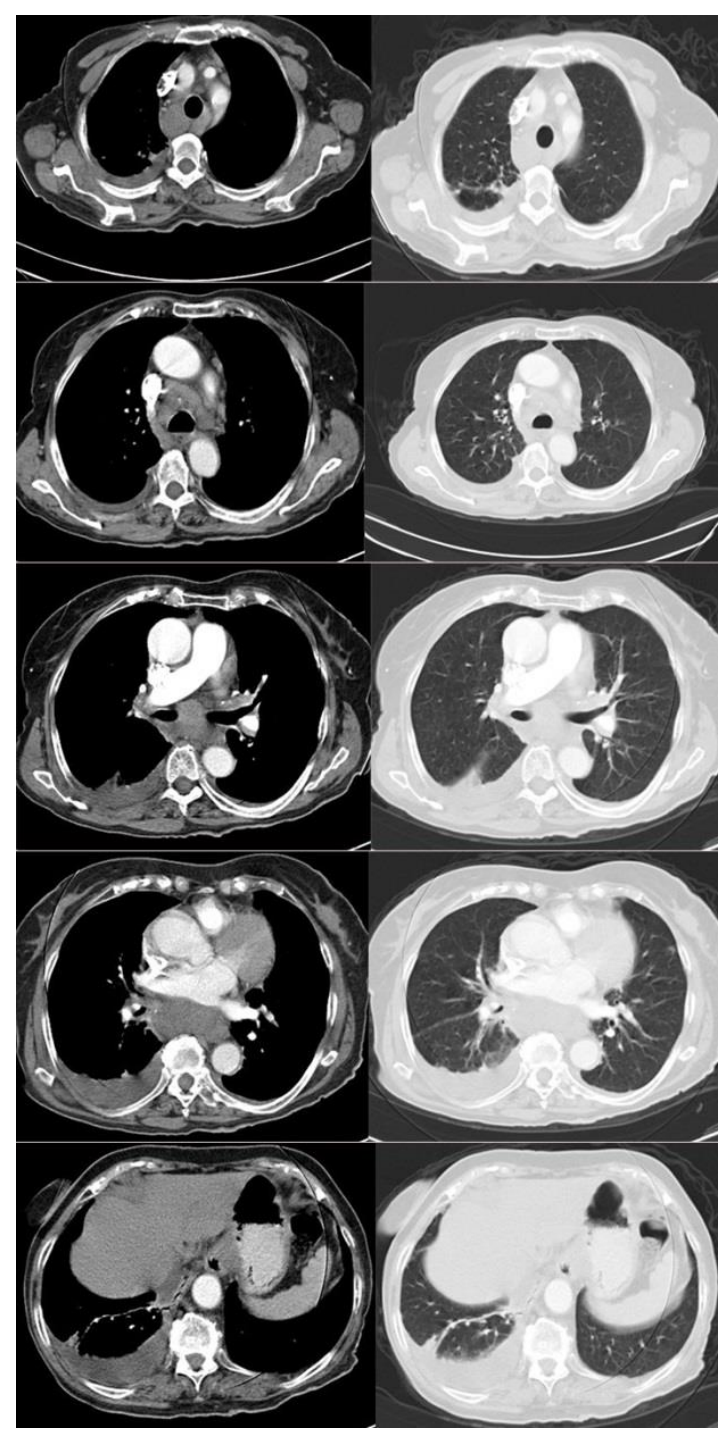

Figure 2: Thoracic CT images.

The patient underwent thoracentesis for pleural effusion in the right hemithorax; it was exudative, fluid culture and mycobacteria culture were negative. Liquid cytology was non-diagnostic. Patient sputum culture of ARB and mycobacteria were requested; results were negative. PPD was $22 \mathrm{~mm}$. FOB was performed; FNAB from subcarinal LAP and lavage from right main bronchus showed benign cytologic findings. Lavage ARB and mycobacteria culture were negative. The pleural effusion in the right hemithorax regressed in the follow up. In the PET CT, many conglomerating lymphadenomegaly (SUV max 7,3) holding FDP were observed in the mediastinum and neck. FNAB was planned from the LAP in the neck but the lesion could not be reached under USG. The patient underwent EBUS; FNAB was obtained from subcarinal and right lower paratracheal LAP. Simultaneous patient control Thorax CT showed a newly developed budding tree in the right lung, newly developing consolidation and pleural effusion in the left lung (Figure 3). 


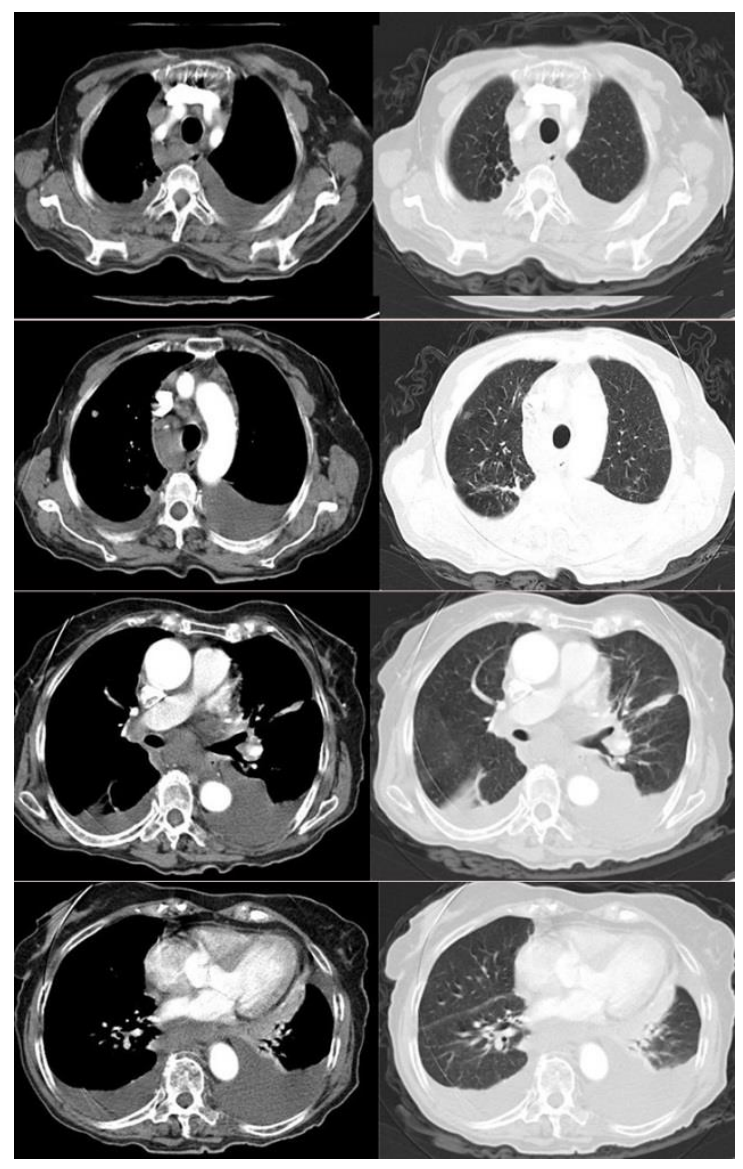

Figure 3: CT images from the follow up.

The patient underwent thoracentesis for a newly developed pleural effusion in the left lung, chylous aspirate was shown (Figure 4). In the fluid triglycerides: $1157 \mathrm{mg} / \mathrm{dl}$, cholesterol: $102 \mathrm{mg} / \mathrm{dl}$, protein:7,3, glucose: 95, ADA: 64. There was no growth in the liquid culture and the culture of ARB and mycobacteria was negative. Liquid cytology was non-diagnostic. The patient's EBUS pathology report showed a necrotizing granulomatous reaction occurring from epithelioid histiocytes.

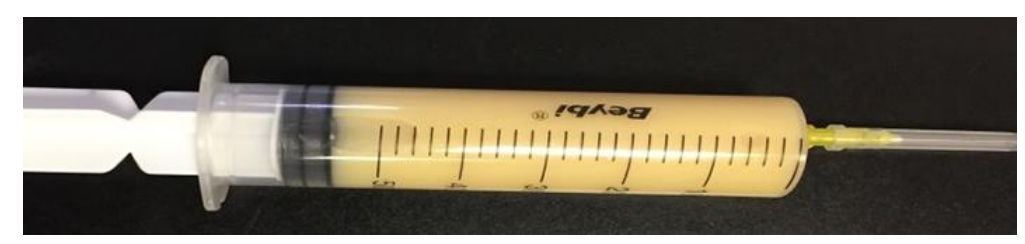

Figure 4: Aspirated fluid in thoracocentesis.

Patient was started a 4-fold anti-TB treatment and conservative treatment for chylothorax was initiated simultaneously. When there was no reduction in chylous fluid in the patient who had been treated with tube thoracostomy, oral feeding was stopped and total parenteral nutrition was initiated (Figure 5). Patients who had daily drainage were treated with pleurodesis twice with talc. There was no increase in effusion after the talk. The patient 
who stopped oral intake had fluid discharge in the follow up, thus talc pleurodesis was performed twice, afterwards thorax tube pulled was pulled out (Figure 6).

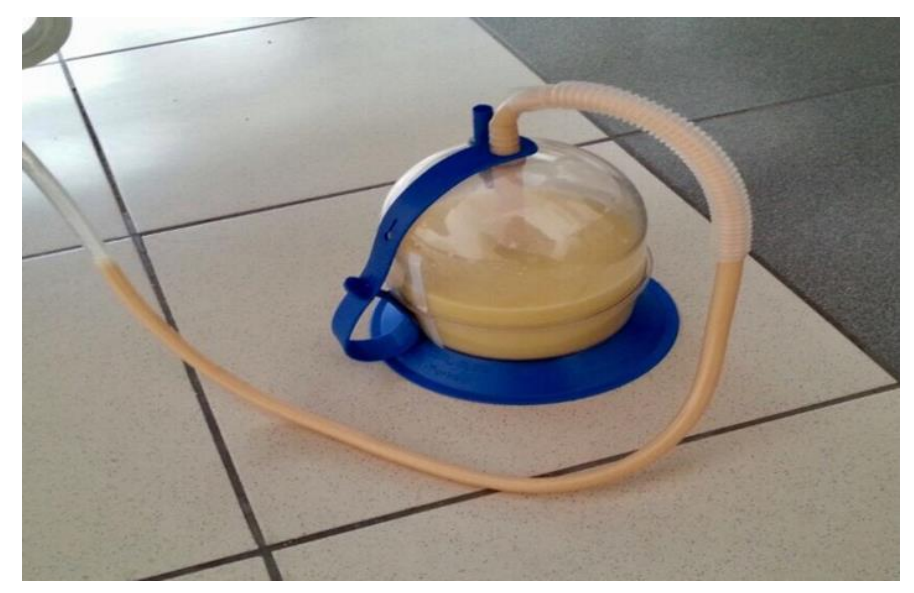

Figure 5: Drainage with tube thoracostomy.

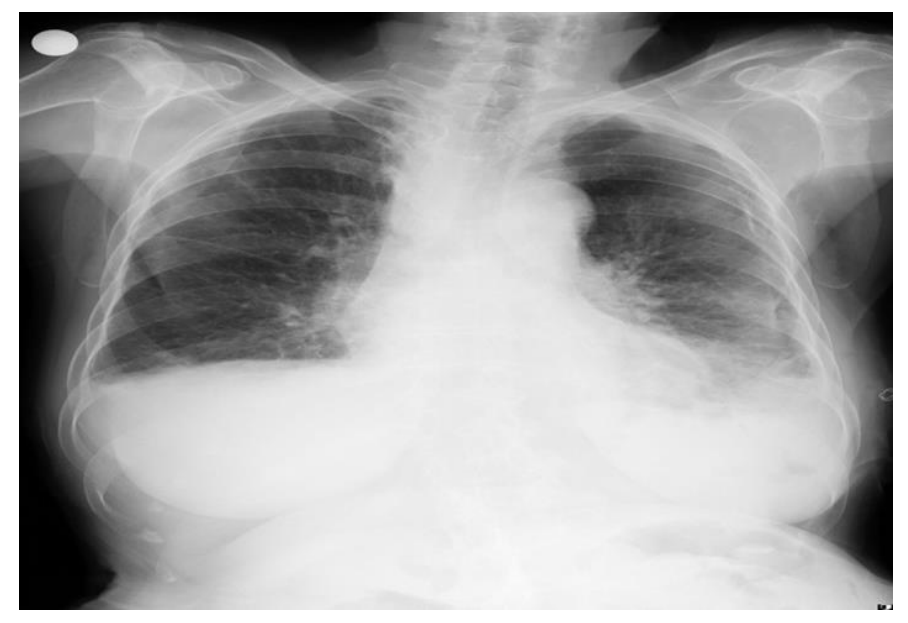

Figure 6: Post treatment postero-anterior graphy.

\section{Discussion}

The ductus thoracicus carries the digested oil from intestines and lymphatic fluid from liver, abdominal wall and lower extremity to the venous system. The chylous fluid is produced in the intestinal system, which gives it a characteristic milky appearance. The chylous fluid contains chylomicron, triglyceride and cholesterol in significant amounts. Other constituents of the lymph are lymphocytes and immunoglobulins. Chylothorax is the leakage of chylous fluid into the pleural space [9]. The first thing to do to put the diagnosis of chylothorax is thoracentesis. Often the fluid taken is milk-like, but it can also be serous or seroangious. Chylous effusions are odorless, exudative and have lymphocyte dominance in the fluid. When the triglyceride level is greater than $110 \mathrm{mg} / \mathrm{dl}$ in the pleural fluid diagnosis can be made, when it is in the range of 50-110 mg/dl lipid electrophoresis is required to demonstrate chylomicrons $[3,10]$. The most common cause of chylothorax is non-Hodgkin lymphoma; the most frequent causes except lymphoma are metastatic cancers and surgical or non-surgical trauma. In a study conducted by Sassoon et al., it has been reported that the development mechanism of chylothorax unrelated to traumatic causes is obliteration of the lymph due to extubation pressure to the ductus thoracicus by the tumor or direct invasion or radiation therapy 
$[11,12]$. Our case was also an oncologic follow-up patient who had been diagnosed with colon cancer before. Primary tumor metastasis was not detected in the lung; yet it was thought to cause chylothorax by direct ductus invasion. Traumatic chylothorax may occur iatrogenicly secondary to percutaneous thoracal interventions resulting in trauma to the left subclavian venous lymphatic drainage or to operational procedures. The blunt or penetrating injury can also damage the lymphatic system; but this is quite rare [13]. The rate of traumatic chylothorax is reported as $2.6 \%$. In our present case, it was considered that the bronchoscopy and EBUS procedures, which were performed for diagnostic purposes, may have caused iatrogenic chylothorax in the patient. In a study done by Tabak et al., granulomatous diseases such as tuberculosis and more rarely sarcoidosis were found to lead to chylothorax [14]. In both diseases, it is suggested that the enlarged mediastinal lymph nodes cause pressure on ductus thoracicus and obstruction caused by erosion and fibrosis lead to chylothorax. In cases of chylothorax associated with tuberculosis; ARB in sputum, fluid or tissues and caseous necrosis are diagnostic. In our case cultures of mycobacteria and ARB observed in sputum and chylous fluid were negative; the ADA level in the pleural effusion was not significant. However, because granulomatous reaction with necrosis in mediastinal LAP biopsy tissue sample has been observed, tuberculosis may be thought to play a role in the etiology of chylothorax in our patient. The main complaints in patients are shortness of breath and chest pain. Treatment should first include diet with medium chained, low fat, high protein and medium chain fatty acids or total parenteral nutrition. Fluid that accumulates in the pleural cavity should be drained by tube thoracostomy and drainage should be closely monitored. It has been reported that the addition of somatostatin and analogs to conservative interventions can increase the efficacy of treatment. Despite the various medical approaches to chylothorax, the most important step in the treatment is to identify the underlying disease. In a study by Hillerdal et al., chylothorax completely disappeared with underlying disease targeting treatments such as steroid therapy in sarcoidosis or medical therapy in heart failure [15]. However, in many diseases such as malignant lymphoma, treatment of the underlying disease may produce good results, but chylothorax may recur and additional measures may be required [16]. In our case, when the etiology of chylothorax is investigated, tuberculous lymphadenitis or direct invasion of the ductus thoracicus by TBC should be considered. But; active malignancy, chemotherapy history and recently recurrent interventional procedures are also involved in the etiology of chylothorax; it should be investigated exactly which of these may have caused the chylothorax.

\section{Acknowledgment}

None

\section{Conflict of Interest}

The author declare that he has no conflict of interest

\section{References}

1. Kamiyoshihara M, Ibe T, Kakegawa S, et al. Late-onset chylothorax after blunt chest trauma at an interval of 20 years: report of a case. Surgery today 38 (2008): 56-58.

2. Doerr CH, Miller DL, Ryu JH. Chylothorax. Semin Respir Crit Care Med 22 (2001): 617-626.

3. Staats BA, Ellefson RD, Budahn LL, et al. The lipoprotein profile of chylous and nonchylous pleural effusions. Mayo Clinic proceedings 55 (1980): 700-704. 
4. Demirhan R, Cevik A, Kucuk HF, et al. Travmatik şilotoraks: Olgu sunumu. Turkish J Thorac Cardiovasc Surg 11 (2003): 50-51.

5. Valentine VG, Raffin TA. The management of chylothorax. Chest 102 (1992): 586-591.

6. Platz A, Simmen HP, Heinzelmann M, et al. Chylothorax after blunt thoracic trauma. Der Chirurg; Zeitschrift fur alle Gebiete der operativen Medizen 66 (1995): 127-130.

7. Ulibarri JI, Sanz Y, Fuentes C, et al. Reduction of lymphorrhagia from ruptured thoracic duct by somatostatin. Lancet 336 (1990): 258.

8. Cho HJ, Kim DK, Lee GD, et al. Chylothorax complicating pulmonary resection for lung cancer: effective management and pleurodesis. The Annals of thoracic surgery 97 (2014): 408-413.

9. Turan O, Ozsan H, Dicle O, et al. A case of chylothorax due to primary renal lymphoma. Case Rep Intern Med 1 (2014): 183-186.

10. Maldonado F, Hawkins FJ, Daniels CE. Pleural fluid characteristics of chylothorax. Mayo Clin Proc 2009;84:129-33. 5.

11. Sassoon CS, Light RW. Chylothorax and pseudochylothorax. Clinics in chest medicine 6 (1985): $163-171$.

12. Strausser JL, Flye MW. Management of nontraumatic chylothorax. The Annals of thoracic surgery 31 (1981): 520-526.

13. Seitelman E, Arellano JJ, Takabe K, et al. Chylothorax after blunt trauma. J Thorac Dis 4 (2012): $327-330$.

14. Tabak L. Şilotoraks psödoşilotoraks. In: Gözü O, Köktürk O (eds). Plevra hastalıkları. Toraks Kitapları. 1. Basım. İstanbul: Turgut Yayıncılık ve Ticaret A.Ş. (2003): 285-294.

15. Hillerdal G. Chylothorax and pseudochylothorax. The European respiratory journal 10 (1997):1157-1162.

16. O'Callaghan AM, Mead GM. Chylothorax in lymphoma: Mechanisms and management. Annals of Oncology 6 (1995): 603-608.

Citation: Kübra Aşık Cansız, Tuncer Tuğ, Suat Konuk, Hacı Ali Kılıçgün, Suphi Aydın. A Case with Tumor, Tuberculosis and Chylothorax. Archives of Clinical and Medical Case Reports 2 (2018): 68-74.

(CC) This article is an open access article distributed under the terms and conditions of the
Creative Commons Attribution (CC-BY) license 4.0

